\title{
Pioglitazone use and risk of bladder cancer: population based cohort study
}

\author{
Marco Tuccori, ${ }^{1,2}$ Kristian B Filion, ${ }^{1,2,3}$ Hui Yin, ${ }^{1}$ Oriana H Yu, 1,4 Robert W Platt, ${ }^{1,2,5,6}$ Laurent Azoulay 1,7
}

${ }^{1}$ Centre for Clinical

Epidemiology, Lady Davis

Institute, Jewish General

Hospital, Montreal, Quebec,

Canada

2Department of Epidemiology,

Biostatistics, and Occupational

Health, McGill University,

Montreal

${ }^{3}$ Division of Clinical

Epidemiology, McGill University

${ }^{4}$ Division of Endocrinology,

Jewish General Hospital,

Montreal

${ }^{5}$ Department of Pediatrics, McGill University, Montreal

${ }^{6}$ Research Institute of the McGill University Health Centre,

Montreal

7Department of Oncology, McGill University

Correspondence to: L Azoulay laurent.azoulay@mcgill.ca

Additional material is published online only. To view please visit the journal online.

Cite this as: BMJ 2016;352:11541 http://dx.doi.org/10.1136/bmj.i1541

Accepted: 29 February 2016

\section{ABSTRACT}

OBJECTIVE

To determine whether pioglitazone compared with other antidiabetic drugs is associated with an increased risk of bladder cancer in people with type 2 diabetes.

\section{DESIGN}

Population based cohort study.

SETTING

General practices contributing data to the United Kingdom Clinical Practice Research Datalink.

\section{PARTICIPANTS}

A cohort of 145806 patients newly treated with antidiabetic drugs between 1 January 2000 and 31 July 2013, with follow-up until 31 July 2014.

\section{MAIN OUTCOME MEASURES}

The use of pioglitazone was treated as a time varying variable, with use lagged by one year for latency purposes. Cox proportional hazards models were used to estimate adjusted hazard ratios with $95 \%$ confidence intervals of incident bladder cancer associated with pioglitazone overall and by both cumulative duration of use and cumulative dose. Similar analyses were conducted for rosiglitazone, a thiazolidinedione not previously associated with an increased risk of bladder cancer.

\section{RESULTS}

The cohort generated 689616 person years of follow-up, during which 622 patients were newly diagnosed as having bladder cancer (crude incidence 90.2 per 100000 person years). Compared with other antidiabetic drugs, pioglitazone was associated with an increased risk of bladder cancer (121.0 v88.9 per 100000 person years; hazard ratio $1.63,95 \%$ confidence interval 1.22 to 2.19 ). Conversely, rosiglitazone was not associated with an increased risk of bladder cancer $(86.2 v 88.9$ per 100000 person

\section{WHAT IS ALREADY KNOWN ON THIS TOPIC}

The association between the use of pioglitazone and bladder cancer is controversial, with studies reporting contradictory findings

Additional observational studies with longer follow-up are needed to assess whether this drug is associated with an increased risk of bladder cancer

\section{WHAT THIS STUDY ADDS}

In this large population based study, the use of pioglitazone was associated with an overall $63 \%$ increased risk of bladder cancer, with the risk increasing with

increasing duration of use and dose

In contrast, the use of rosiglitazone was not associated with an increased risk, with no evidence of a duration-response or dose-response relation

These findings suggest that the association observed with pioglitazone is likely to be a drug specific and not a class effect

years; $1.10,0.83$ to 1.47$)$. Duration-response and dose-response relations were observed for pioglitazone but not for rosiglitazone.

\section{CONCLUSION}

The results of this large population based study indicate that pioglitazone is associated with an increased risk of bladder cancer. The absence of an association with rosiglitazone suggests that the increased risk is drug specific and not a class effect.

\section{Introduction}

Pioglitazone, an antidiabetic drug belonging to the thiazolidinedione class, has been shown to improve glycaemic levels in people with type 2 diabetes. ${ }^{1}$ However, in 2005 the PROactive randomised controlled trial unexpectedly showed an imbalance in the number of cases of bladder cancer with pioglitazone compared with placebo. ${ }^{2}$ In contrast, this imbalance was never observed in randomised controlled trials of rosiglitazone, the other approved drug belonging to the thiazolidinedione class. ${ }^{13}$

The findings of the PROactive trial were subsequently corroborated in some, ${ }^{4-10}$ but not all, observational studies. ${ }^{11-19}$ Indeed, in the five year interim analysis of a large observational study using the Kaiser Permanente Northern California database, ${ }^{4}$ the use of pioglitazone for 24 months or more was associated with an increased risk of bladder cancer (hazard ratio 1.4, 95\% confidence interval 1.03 to 2.0). However, in the final analysis of the Kaiser Permanente Northern California study, which used the same cohort ${ }^{4}$ with follow-up extended to 10 years, the use of pioglitazone was no longer significantly associated with an increased risk of bladder cancer in a duration-response fashion. ${ }^{20}$ These null findings are also consistent with those of another large multicohort study. ${ }^{19}$ The apparent heterogeneity in this literature may be due to methodological limitations, such as the inclusion of prevalent users, ${ }^{5610-1418}$ time lag bias, ${ }^{15}$ immortal time bias, ${ }^{101418}$ and no consideration of disease latency. 810121718

Given these discrepant findings, the methodological shortcoming of previous studies examining this association, and the apparent loss of an association in studies with longer follow-up, ${ }^{20}$ additional studies are needed to investigate further the association between pioglitazone and bladder cancer. In a large, population based study we assessed the association between the use of pioglitazone and bladder cancer in people with type 2 diabetes.

\section{Methods}

Data source

This study was conducted using the United Kingdom Clinical Practice Research Datalink (CPRD). This 
database contains the complete primary care medical record of more than 13 million people. ${ }^{21}$ The Read code classification is used to record medical diagnoses and procedures, and a coded drug dictionary based on the UK Prescription Pricing Authority Dictionary is used to record prescriptions. The CPRD collects information on anthropometric variables such as body mass index and lifestyle variables such as smoking. Data collected in the CPRD have been previously validated and shown to be of high quality. ${ }^{2223}$ Furthermore, cancer diagnoses have been found to be highly consistent with those recorded in the UK national cancer data repository. ${ }^{24}$

\section{Study population \\ Base cohort}

We assembled a base cohort composed of all people newly treated for type 2 diabetes, defined as receiving a first ever prescription for a non-insulin antidiabetic drug (metformin, sulfonylureas, prandial glucose regulators, thiazolidinediones, acarbose, dipeptidyl peptidase-4 (DPP-4) inhibitors, glucagon-like peptide (GLP-1) agonists, sodium-glucose cotransporter-2 (SGLT2) inhibitors) between 1 January 1988 and 31 July 2013. Patients were required to be at least 40 years of age and to have at least one year of CPRD medical history before that first prescription. We excluded patients prescribed insulin any time before their first non-insulin antidiabetic prescription (as these may represent those with an advanced form of type 2 diabetes), and patients with a diagnosis of gestational diabetes and polycystic ovary syndrome (as these are other indications for metformin).

\section{Study cohort}

Using the base cohort, we identified all patients who initiated a new antidiabetic drug class on or after 1 January 2000 (the year pioglitazone and rosiglitazone entered the UK market) until 31 July 2013. These patients included those newly treated with an antidiabetic drug class, as well as those who switched to or added-on an antidiabetic drug class not previously used in their treatment. Cohort entry was defined by the date of this new prescription. We excluded all patients with a diagnosis of bladder cancer (including malignant, in situ, and benign lesions) at any time before cohort entry, as well as those with less than one year of follow-up after cohort entry. The latter was necessary for latency considerations, as short term drug use are unlikely to cause incident bladder cancer.

All patients were followed from the year after cohort entry until a first ever diagnosis of bladder cancer (malignant and in situ), or censored on death from any cause, end of registration with the general practice, or end of the study period (31 July 2014), whichever occurred first.

\section{Use of thiazolidinediones}

In the models we entered the use of thiazolidinediones as a time varying variable and classified it according to one of the four mutually exclusive categories: pioglitazone use, rosiglitazone use, pioglitazone and rosiglitazone use (mainly switchers), and no thiazolidinedione use. Patients were considered unexposed to thiazolidinediones until the time of the first thiazolidinedione prescription and thereafter considered exposed, after accounting for a one year lag period. This lag period was necessary to take into account a latency time window and to minimise possible detection bias around the time of treatment initiation. This was considered the primary exposure definition.

In secondary analyses, we determined whether there was a duration-response and dose-response relation between pioglitazone and incidence of bladder cancer. The duration-response relation was assessed in terms of cumulative duration of use, which was defined, in a time dependent fashion, as the total number of years of use, calculated by summing the durations of all prescriptions received between cohort entry and the time of the event. This variable was then classified using the same categories used in the interim analysis of the Kaiser Permanente Northern California study ${ }^{4}:<1$ year, 1 to 2 years, and $>2$ years of use. We also assessed cumulative duration on a continuous scale using a restricted cubic spline model with five knots. ${ }^{25}$ Dose-response was assessed in terms of cumulative dose, which was calculated in a time dependent fashion as the sum of all doses received up until the date of the event. This variable was also categorised using the same cut-offs used in previous studies ${ }^{45}$ : $\leq 10500 \mathrm{mg}, 10501-28000 \mathrm{mg}$, and $>28000 \mathrm{mg}$. We assessed the linear trend for cumulative duration of use and dose by considering these variables as continuous in the models.

For comparison purposes we also assessed whether there was a duration-response and dose-response relation with rosiglitazone, in terms of cumulative duration of use (categorically ( $<1$ year, 1 to 2 years, and $>2$ years) and continuously using restricted cubic spline modelling) and cumulative dose (categorised on the basis of the distribution of use in thirds in the cohort).

\section{Potential confounders}

All models were adjusted for several variables measured at cohort entry: age, sex, year of cohort entry, body mass index $\left(<30 \mathrm{~kg} / \mathrm{m}^{2}, \geq 30 \mathrm{~kg} / \mathrm{m}^{2}\right.$, unknown; last measure before cohort entry), smoking status (ever, never, unknown), alcohol related disorders (based on diagnoses for alcohol misuse, alcoholic cirrhosis of the liver, alcoholic hepatitis and failure, and other related disorders), haemoglobin A1c ( $\leq 7.4 \%,>7.4 \%$, unknown; last recorded value before cohort entry), duration of treated diabetes (defined as the time between the first ever non-insulin prescription and cohort entry), previous bladder conditions (cystitis and bladder stones), history of cancer (other than non-melanoma skin cancer), presence of at least one urine protein test in the year before cohort entry, and Charlson comorbidity score $^{26}$ (adapted so as not to include previous cancer, to avoid duplicate adjustment).

\section{Statistical analysis}

Descriptive statistics were used to summarise the characteristics of pioglitazone users, rosiglitazone users, and non-thiazolidinedione users at cohort entry. We 
calculated crude incidence rates of bladder cancer, with 95\% confidence intervals based on the Poisson distribution overall and for each exposure category.

Time dependent Cox proportional hazards models, with duration of follow-up as the underlying time axis, were used to estimate adjusted hazard ratios and 95\% confidence intervals for bladder cancer associated with the use of pioglitazone compared with no thiazolidinedione use. We also conducted two secondary analyses to assess whether there were duration-response and dose-response relations with pioglitazone and risk of bladder cancer in terms of cumulative duration of use and cumulative dose (as defined previously). Identical analyses were done for rosiglitazone. For all models we used the model proposed by Fine and Gray to account for competing risks due to death from any cause. ${ }^{27}$ We examined the Schoenfeld residuals for the time fixed covariates and found no important departures from the proportional hazards assumption.

\section{Sensitivity analyses}

We conducted nine sensitivity analyses to assess the robustness of our findings. Firstly, given uncertainties related to the latency time window, we repeated the primary analysis with lag periods of zero and two years. Secondly, we repeated the primary analysis after considering a stricter definition for drug use based on receiving at least four prescriptions within a 12 month window. Thirdly, we repeated the analysis after excluding patients with a history of bladder conditions at any time before cohort entry and censoring on a new diagnosis during follow-up. Fourthly, we repeated the primary analysis after additionally censoring on a new diagnosis of benign bladder lesions, in situ bladder cancer, liver failure, and heart failure (the last two were also additional exclusion criteria). Indeed, thiazolidinediones are contraindicated or not recommended for the two last conditions, the presence of which may lead to thiazolidinedione discontinuation or may influence treatment decisions. Fifthly, in 2011 several regulatory actions were issued because of the potential association between pioglitazone and bladder cancer. ${ }^{28}$ We performed a sensitivity analysis censoring follow-up to 31 December 2010, as it is possible that patients starting or continuing pioglitazone after that date may have been more carefully screened for bladder cancer. Sixthly, we repeated the primary analysis using multiple imputation for variables with missing values (that is, body mass index, smoking, and haemoglobin A1c). ${ }^{29} 30$ Seventhly, we additionally adjusted the models for the time dependent use of other antidiabetic drugs (metformin, sulfonylureas, incretin based drugs (GLP-1 analogues or DPP-4 inhibitors), insulin, and other oral hypoglycaemic drugs) during follow-up, lagged by one year for latency considerations. Eighthly, to account for potential time dependent confounding during the 14.5 year study period, we repeated the primary analysis using a marginal structural Cox proportional hazards model with inverse probability of treatment and censoring weighting (see the supplementary file for a detailed description of this method). Finally, we assessed the strength of an unmeasured confounder needed to move the estimated hazard ratio to the null using the "rule out" method proposed by Schneeweiss. ${ }^{31}$

\section{Head to head comparison of pioglitazone with rosiglitazone}

To assess further whether an association between pioglitazone and bladder cancer is a drug specific compared with a class effect, we conducted two additional analyses that directly compared pioglitazone with rosiglitazone. In the first approach, we contrasted the use of pioglitazone with the use of rosiglitazone by repeating our primary analysis with the latter as the reference category. In the second approach, we used the study cohort to assemble a subcohort of patients starting pioglitazone or rosiglitazone between 1 January 2000 and 31 July 2013, with follow-up until 31 July 2014. As with the primary analysis, all patients were required to have at least one year of follow-up after their first prescription for a thiazolidinedione. Consequently, cohort entry was set as the year after the first thiazolidinedione prescription during the study period. All patients were followed until a first ever diagnosis of bladder cancer, or censored on death from any cause, switching to another thiazolidinedione, end of registration with the general practice, or end of the study period, whichever occurred first. The model was adjusted for high dimensional propensity scores, ${ }^{32}$ which included the prespecified variables listed previously along with another 500 empirically defined variables measured at the time of the first thiazolidinedione prescription. All analyses were conducted with SAS version 9.4 (SAS Institute, Cary, NC).

\section{Patient involvement}

No patients were involved in setting the research question or the outcome measure, nor were they involved in developing plans for design or implementation of the study. No patients were asked to advise on interpretation or writing up of results. There are no plans to disseminate the results of the research to study participants or the relevant patient community.

\section{Results}

A total of 145806 patients met the study inclusion criteria (see supplementary figure 1). Overall, the cohort was followed for a mean of 4.7 (SD 3.4) years, generating 689616 person years of follow-up. Overall, 622 patients received a diagnosis of bladder cancer during follow-up, yielding a crude incidence rate of 90.2 (95\% confidence interval 83.2 to 97.6) per 100000 person years. Among patients with an event, the median time between cohort entry and an incident diagnosis of bladder cancer was 4.4 (interquartile range 2.5-6.5) years.

Table 1 presents the characteristics of the cohort overall and stratified by pioglitazone users versus non-thiazolidinedione users at baseline. Compared with non-thiazolidinedione users, pioglitazone users were less likely to be obese but more likely to have increased haemoglobin A1c levels, to have undergone urine protein testing before cohort entry, had a longer duration of 
treated diabetes, and were more likely to have previous bladder conditions. Pioglitazone users were also more likely to have used sulfonylureas and less likely to have received metformin compared with non-users of thiazolidinediones. The baseline characteristics of rosiglitazone users are similar and shown in supplementary table 1.

Table 2 shows the results of the primary and secondary analyses for pioglitazone. Compared with no thiazo-

\begin{tabular}{|c|c|c|c|}
\hline Characteristics & $\begin{array}{l}\text { Entire cohort } \\
(n=145806)\end{array}$ & $\begin{array}{l}\text { Pioglitazone* } \\
(\mathrm{n}=921)\end{array}$ & $\begin{array}{l}\text { No pioglitazone } \\
(\mathrm{n}=142 \mathrm{258})\end{array}$ \\
\hline Male & $82824(56.8)$ & $543(59.0)$ & $81114(56.8)$ \\
\hline Mean (SD) age (years) & $63.7(11.7)$ & $64.6(10.6)$ & $63.7(11.7)$ \\
\hline \multicolumn{4}{|l|}{ Year of cohort entry: } \\
\hline 2000 & $8167(5.6)$ & Suppressed $\ddagger$ & $7970(5.6)$ \\
\hline 2001 & $9445(6.5)$ & $126(13.7)$ & $8938(6.3)$ \\
\hline 2002 & $9604(6.6)$ & $120(13.0)$ & $9224(6.5)$ \\
\hline 2003 & $10393(7.1)$ & 114 (12.4) & $10040(7.0)$ \\
\hline 2004 & $12141(8.3)$ & $138(15.0)$ & 11624 (8.1) \\
\hline 2005 & $11683(8.0)$ & $106(11.5)$ & $11273(7.9)$ \\
\hline 2006 & $11126(7.6)$ & $84(9.1)$ & $10810(7.6)$ \\
\hline 2007 & $11657(8.0)$ & $64(7.0)$ & $11477(8.0)$ \\
\hline 2008 & $11731(8.1)$ & $53(5.8)$ & $11664(8.2)$ \\
\hline 2009 & $12445(8.5)$ & $50(5.4)$ & $12391(8.7)$ \\
\hline 2010 & $12035(8.3)$ & $36(3.9)$ & $11995(8.4)$ \\
\hline 2011 & $10659(7.3)$ & $14(1.5)$ & $10645(7.5)$ \\
\hline 2012 & $10110(6.9)$ & $9(1.0)$ & $10101(7.1)$ \\
\hline 2013 & $4610(3.2)$ & Suppressed $\neq$ & $4606(3.2)$ \\
\hline \multicolumn{4}{|l|}{ Body mass index: } \\
\hline$<30 \mathrm{~kg} / \mathrm{m}^{2}$ & $67621(46.4)$ & $479(52.0)$ & $66152(46.3)$ \\
\hline$\geq 30 \mathrm{~kg} / \mathrm{m}^{2}$ & $76627(52.6)$ & $433(47.0)$ & $75076(52.6)$ \\
\hline Unknown & $1558(1.1)$ & $9(1.0)$ & $1530(1.1)$ \\
\hline \multicolumn{4}{|l|}{ Smoking: } \\
\hline Ever & $85032(58.3)$ & $523(56.8)$ & $83342(58.4)$ \\
\hline Never & $57283(39.3)$ & $384(41.7)$ & $55982(39.2)$ \\
\hline Unknown & $3491(2.4)$ & $14(1.5)$ & $3434(2.4)$ \\
\hline Alcohol related disorders & $15491(10.6)$ & $80(8.7)$ & $15240(10.7)$ \\
\hline \multicolumn{4}{|l|}{ Haemoglobin A1c: } \\
\hline$\leq 7.4 \%$ & $27209(18.7)$ & $148(16.1)$ & $26793(18.8)$ \\
\hline$>7.4 \%$ & $68309(46.9)$ & $537(58.3)$ & $66485(46.6)$ \\
\hline Unknown & $50288(34.5)$ & $236(25.6)$ & $49480(34.7)$ \\
\hline Mean (SD) duration of treated diabetes (years) & $0.3(1.6)$ & $4.2(4.6)$ & $0.3(1.3)$ \\
\hline Previous bladder conditions & $13755(9.4)$ & $113(12.3)$ & $13415(9.4)$ \\
\hline Cancer & $13908(9.5)$ & $76(8.3)$ & $13646(9.6)$ \\
\hline Urine protein test & $62729(43.0)$ & $491(53.3)$ & $61072(42.8)$ \\
\hline Mean (SD) Charlson comorbidity score & $2.0(1.3)$ & $2.2(1.4)$ & $2.0(1.3)$ \\
\hline \multicolumn{4}{|l|}{ Previous antidiabetic drug use§: } \\
\hline Metformin & $122843(84.3)$ & $497(54.0)$ & $120765(84.6)$ \\
\hline Sulfonylureas & $31825(21.8)$ & $433(47.0)$ & $30217(21.2)$ \\
\hline Pioglitazone & $921(0.6)$ & $921(100.0)$ & $0(0.0)$ \\
\hline Rosiglitazone & $2127(1.5)$ & $0(0.0)$ & $0(0.0)$ \\
\hline Incretin based drugs & $375(0.3)$ & $0(0.0)$ & $375(0.3)$ \\
\hline Insulins & $1467(1.0)$ & $14(1.5)$ & $1435(1.0)$ \\
\hline Others & $1406(1.0)$ & $45(4.9)$ & $1217(0.9)$ \\
\hline
\end{tabular}

Patients exposed to rosiglitazone alone or together with pioglitazone are not displayed in the table.

*Pioglitazone only users at cohort entry.

tNo use of any thiazolidinedione at cohort entry.

\#Numbers $<5$ are not displayed, following the confidentiality policies of the Clinical Practice Research Datalink.

IIncluding myocardial infarction, congestive heart failure, peripheral vascular disease, chronic pulmonary

disease, cerebrovascular disease, dementia, peptic ulcer disease, diabetes related chronic complications, connective tissue disease, mild liver disease, hemiplegia or paraplegia, renal disease, moderate to severe liver disease, AIDS. Adapted to exclude cance

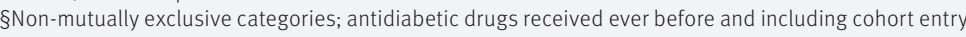

lidinedione use, the use of pioglitazone was associated with an increased risk of incident bladder cancer (121.0 $v 88.9$ per 100000 person years; adjusted hazard ratio $1.63,95 \%$ confidence interval 1.22 to 2.19). A duration-response relation was observed $(\mathrm{P}<0.01$ for trend) with use of pioglitazone for more than two years associated with an increased risk of bladder cancer (adjusted hazard ratio $1.78,95 \%$ confidence interval 1.21 to 2.64 ). In the restricted cubic spline analysis, the risk of bladder cancer was increased after 1.8 years of pioglitazone use, and continued to increase with longer durations of use, although this did not achieve statistical significance owing to a relatively small number of events among patients with longer duration of use (fig 1). A dose-response relation was also present $(\mathrm{P}=0.01$ for trend), with cumulative doses less than $10500 \mathrm{mg}$ (adjusted hazard ratio $1.63,95 \%$ confidence interval 1.02 to 2.60 ) and more than $28000 \mathrm{mg}$ (1.70, 1.04 to 2.78 ) being associated with an increased risk of bladder cancer.

Overall, the use of rosiglitazone was not associated with an increased risk of incident bladder cancer (86.2 $v 88.9$ per 100000 person years, adjusted hazard ratio $1.10,95 \%$ confidence interval 0.83 to 1.47 ; see supplementary table 2). Similarly, there was no evidence of a duration-response relation in terms of cumulative duration of use when it was classified as a categorical variable $(\mathrm{P}=0.7$ for trend; see supplementary table 2$)$ or when it was considered as a continuous variable (see supplementary figure 2). Finally, there was no evidence of a dose-response relation in terms of cumulative dose ( $\mathrm{P}=0.7$ for trend; see supplementary table 2 ).

\section{Sensitivity analyses}

The results of the sensitivity analyses are summarised in figure 2 and presented in supplementary tables 3 and 4. In all sensitivity analyses, the use of pioglitazone was consistently associated with an increased risk of bladder cancer, with adjusted hazard ratios ranging between 1.46 and 1.76. In contrast, the use of rosiglitazone was not associated with an increased risk of bladder cancer, with adjusted hazard ratios ranging between 1.01 and 1.16 and all estimates accompanied by $95 \%$ confidence intervals that included unity. Supplementary Figure 3 shows the exposure-confounder and confounder-disease associations (right of the curve) necessary to reduce the observed hazard ratio of 1.63 down to the null.

\section{Head to head comparison of pioglitazone with rosiglitazone}

Table 3 presents the results of the comparisons between pioglitazone and rosiglitazone. In the first of these analyses (main model), pioglitazone use compared with rosiglitazone use was associated with an increased risk of bladder cancer (adjusted hazard ratio 1.48, 95\% confidence interval 1.01 to 2.16). Similar findings were observed in the second analysis conducted within the thiazolidinedione subcohort (hazard ratio adjusted for high dimensional propensity scores 1.46 , 95\% confidence interval 0.94 to 2.27; see supplementary figure 4 and supplementary table 5 for cohort description). 


\begin{tabular}{|c|c|c|c|c|c|}
\hline No thiazolidinedione & 497 & 558924 & 88.9 (81.3 to 97.1) & 1.00 (Reference) & 1.00 (Reference) \\
\hline Pioglitazone & 54 & 44618 & 121.0 (90.9 to 157.9) & 1.68 (1.26 to 2.24$)$ & 1.63 (1.22 to 2.19) \\
\hline $1-2$ years & 14 & 11583 & 120.9 (66.1 to 202.8) & 1.70 (1.00 to 2.91$)$ & 1.66 (0.97 to 2.84$)$ \\
\hline$>2$ years & 29 & 21004 & 138.1 (92.5 to 198.3$)$ & $1.84(1.25$ to 2.71$)$ & 1.78 (1.21 to 2.64$)$ \\
\hline $\mathrm{P}$ trend & & & & $<0.01$ & $<0.01$ \\
\hline \multicolumn{6}{|l|}{ Cumulative dose: } \\
\hline$\leq 10500 \mathrm{mg}$ & 18 & 15646 & 115.0 (68.2 to 181.8$)$ & 1.66 (1.04 to 2.67$)$ & 1.63 (1.02 to 2.60$)$ \\
\hline \multicolumn{6}{|c|}{$\begin{array}{l}\text { *Users of rosiglitazone and users of combination of pioglitazone and rosiglitazone are not displayed in the table, but were considered in the regression } \\
\text { model for proper estimation of treatment effects. } \\
\text { †Per } 100000 \text { person years. } \\
\text { ¥Adjusted for age, year of cohort entry, sex, alcohol related disorders, smoking status, obesity, haemoglobin A1c, previous cancer, bladder conditions, } \\
\text { Charlson comorbidity score, duration of treated diabetes, and urine protein testing. } \\
\text { १No use of pioglitazone or rosiglitazone. }\end{array}$} \\
\hline
\end{tabular}

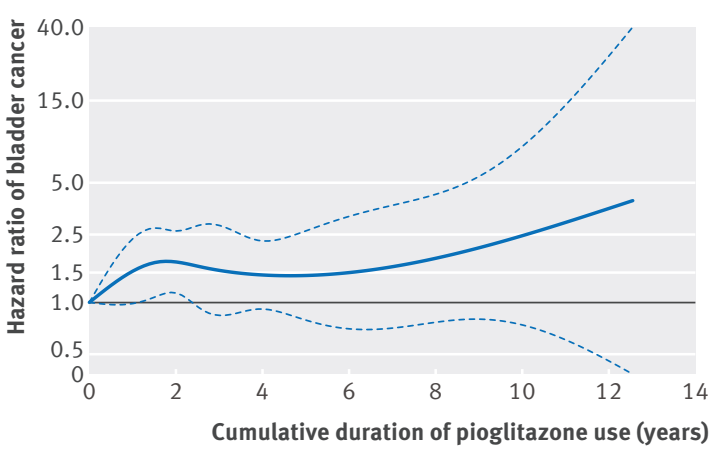

Fig 1 | Smooth restricted spline curve of adjusted hazard ratio of bladder cancer (solid line) and $95 \%$ confidence limits (dashed lines) as function of cumulative duration of pioglitazone use

\section{Discussion}

In this large population based cohort study with up to 14.5 years of follow-up, pioglitazone was associated with an overall 63\% increased risk of incident bladder cancer. There was also evidence of a duration-response and dose-response relations. In contrast, rosiglitazone was not associated with an increased risk of bladder cancer either overall or by cumulative duration of use and dose. Our findings remained consistent in several sensitivity analyses.

\section{Strengths and weaknesses of this study}

This study has several strengths. Firstly, we assembled a large population based cohort of patients newly treated with antidiabetic drugs and followed for up to 14.5 years, thus enabling the identification of a substantial number of patients with bladder cancer. Secondly, the inclusion of new users eliminated biases related to prevalent users. ${ }^{33}$ Thirdly, we considered a lag period to account for a minimum latency between use of thiazolidinediones and the development of bladder cancer. Fourthly, we defined exposure in a time dependent fashion, thereby eliminating immortal time bias. ${ }^{34}$ Fifthly, all analyses took into account competing risks due to deaths from any cause, an important consideration given the cardiovascular risk reported for thiazolidinediones in previous studies. ${ }^{1}$ Finally, the results remained consistent in several sensitivity analyses, thus confirming the robustness of our findings.

This study has some limitations. Firstly, residual confounding from unmeasured variables (eg, diet, physical activity, occupational exposure, pelvic radiation, family history of cancer, and race/ethnicity) is possible. However, the rule out method ${ }^{31}$ shows that a hypothetical unmeasured confounder would need to be strongly associated with both the exposure (odds ratio $>3.7$ ) and the outcome (relative risk $>5.0$ ) to move the point estimate down to the null. As the aforementioned variables are modestly associated with the outcome and it is unclear if they are associated with the exposure, we do not believe that residual confounding is a likely explanation for the observed association. Secondly, misclassification of drug use is possible, since the CPRD records prescriptions written by general practitioners and not those written by specialists. However, although some specialists may have been responsible for patients starting thiazolidinediones, general practitioners are likely to have been those prescribing repeat prescriptions for these drugs. Thus, misclassification is likely to minimal and, if present, would lead to an underestimation of the association. Finally, although cancers of the urinary tract have been shown to be well recorded in the CPRD, ${ }^{24}$ misclassification is possible. However, we expect this potential misclassification to be nondifferential between patients using the different antidiabetic drugs included in the study.

\section{Comparison with other studies}

Several observational studies have investigated the association between pioglitazone and bladder cancer. $^{4-19}$ Overall, these studies have generated conflicting findings, with seven reporting statistically significant 


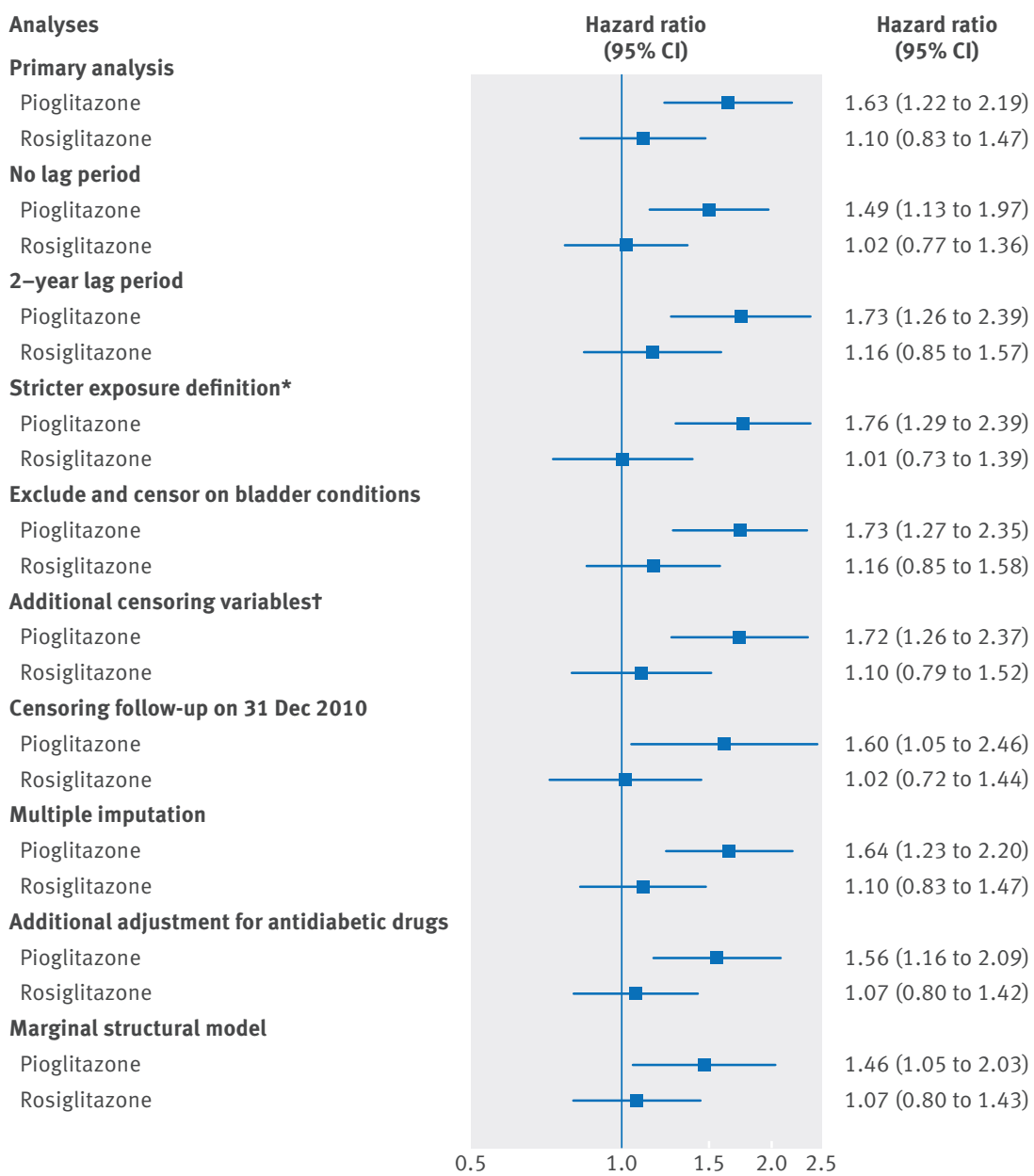

Fig 2 | Forest plot for primary and sensitivity analyses displaying adjusted hazard ratios for association between pioglitazone use and rosiglitazone use and risk of bladder cancer. * Receiving at least four prescriptions within a 12 month moving window. †Benign bladder lesions, in situ bladder cancer, heart failure, and liver failure

increased risks ${ }^{4-10}$ (ranging from 20\% to 225\%) and nine reporting null associations. ${ }^{11-19}$ The discrepancy between these studies is likely due to certain methodological shortcomings. Indeed, in three studies, ${ }^{101418}$ the definition for drug use may have introduced immortal time bias, a bias resulting from the misclassification of unexposed person time as exposed person time, which may have led to a spurious underestimate of the association. In another study, ${ }^{15}$ time lag bias was introduced by comparing pioglitazone with insulin, the latter being a drug typically used at a more advanced stage of the disease, where the risk of cancer, including bladder cancer, may be higher. Prevalent users of antidiabetic drugs were included in 11 studies, ${ }^{4-611-1418-20}$ which can be problematic in this context given the relatively rapid onset of bladder cancer after starting pioglitazone. Finally, in five studies, 810121718 a minimum time between starting pioglitazone and the diagnosis of bladder cancer was not considered in the analyses, an important consideration given the latency of bladder cancer.

Our findings are consistent with those of the recently published Insulin Resistance Intervention after Stroke (IRIS) trial, which randomised 3895 people without diabetes to either pioglitazone or placebo. ${ }^{35}$ After a median follow-up of 4.8 years, pioglitazone was associated with a decreased risk of a composite endpoint of stroke or myocardial infarction (hazard ratio 0.76, 95\% confidence interval 0.62 to 0.93 ). However, there was an increased number of bladder cancer events in the pioglitazone group compared with the placebo group (12 $(0.6 \%) v 8(0.4 \%)$, respectively). ${ }^{35}$ This imbalance was observed despite efforts to exclude patients with a history of or at high risk of bladder cancer (that is, defined by the presence of macroscopic haematuria, use of cyclophosphamide, or previous radiation to the pelvis). ${ }^{36}$ Thus, although this imbalance did not reach statistical significance, ${ }^{35}$ it mirrors the imbalance observed in the PROactive triall ${ }^{2}$ and is consistent with the effect size reported in our study.

\section{Biological plausibility and implications}

The biological plausibility of a rapid development of bladder cancer after starting pioglitazone has been debated, since many events observed in the PROactive trial occurred within one year of starting treatment. ${ }^{37}$ It is possible that these were prevalent cases and not attributable to pioglitazone, ${ }^{37}$ or promoted by pioglitazone in patients susceptible to developing bladder cancer. ${ }^{38}$ In our study, the use of a one year lag period

\begin{tabular}{|c|c|c|c|c|c|}
\hline Exposure & $\begin{array}{l}\text { No of } \\
\text { patients* }\end{array}$ & $\begin{array}{l}\text { No of } \\
\text { events }\end{array}$ & $\begin{array}{l}\text { Person } \\
\text { years }\end{array}$ & $\begin{array}{l}\text { Incidence rate } \\
(95 \% \mathrm{Cl}) \dagger\end{array}$ & $\begin{array}{l}\text { Adjusted hazard } \\
\text { ratio }(95 \% \mathrm{Cl})\end{array}$ \\
\hline Rosiglitazone & - & 56 & 64990 & 86.2 (65.1 to 111.9) & 1.00 (Reference) \\
\hline Pioglitazone & - & 54 & 44618 & 121.0 (90.9 to 157.9) & $1.48(1.01$ to 2.16$)$ \\
\hline \multicolumn{6}{|c|}{ Thiazolidinedione subcohort to cohort analysis§ **: } \\
\hline Pioglitazone & 10591 & 52 & 44080 & 118.0 (88.1 to 154.7$)$ & 1.46 (0.94 to 2.27) \\
\hline \multicolumn{6}{|c|}{$\begin{array}{l}\text { *Number of patients in main analysis is not displayed as exposure was defined in a time dependent fashion. } \\
\text { †Per } 100000 \text { person years. } \\
\text { fUsers of pioglitazone to rosiglitazone combinations and no thiazolidinedione users are not displayed in the table, but were considered in the regression } \\
\text { model for proper estimation of treatment effects } \\
\text { IAdjusted for age, year of cohort entry, sex, alcohol related disorders, smoking status, obesity, haemoglobin A1c, previous cancer, bladder conditions, } \\
\text { Charlson comorbidity score, duration of treated diabetes, and urine protein testing. } \\
\text { §Two bladder cancer events were excluded from the pioglitazone group owing to trimming related to non-overlapping propensity score distributions. } \\
\text { **Adjusted for high dimensional propensity score fifths. }\end{array}$} \\
\hline
\end{tabular}


ensured that all bladder cancer events had to occur at least one year after starting treatment. However, in sensitivity analyses, removing the lag period attenuated the hazard ratio $(1.49,95 \%$ confidence interval 1.13 to 1.97), whereas applying a two year lag period increased the hazard ratio $(1.73,1.26$ to 2.39$)$. Moreover, when assessed in a restricted cubic spline model, the risk tended to increase with longer durations of use. Taken together, our findings do not rule out a tumour promoting effect but also suggest that the risk may increase with longer use.

An important finding of our study is the absence of an association between rosiglitazone and bladder cancer. It is important to note that both pioglitazone and rosiglitazone entered the UK market the same year (2000) and both were intended for the same target population. ${ }^{39}$ Given their similarities, it is unlikely that confounding by indication or detection bias can explain the association observed with pioglitazone. In the head to head comparison, pioglitazone was associated with close to a $50 \%$ increased risk of bladder cancer compared with rosiglitazone. Of note, although the biological mechanism for pioglitazone induced bladder cancer is not clear, this imbalance in the risk of bladder cancer between these two thiazolidinediones could likely be explained by pharmacological differences. Indeed, unlike rosiglitazone, which is selective for the peroxisome proliferator activated receptor (PPAR) $\gamma$, pioglitazone has a dual PPAR $\alpha / \gamma$ activity. ${ }^{4041}$ This is particularly important, as PPAR $\alpha / \gamma$ activation in rat models has been shown to increase the expression of carcinogenic biomarkers in the bladder, which has not been observed with the selective activation of PPAR $\gamma .42-44$ Although differences in PPAR activity are possible explanations for the observed association, additional studies are needed to better understand the biological mechanism behind the possible pioglitazone specific effect on the bladder.

\section{Conclusions}

The use of pioglitazone is associated with an increased risk of bladder cancer, which varies in a duration dependent and dose dependent fashion. In contrast, rosiglitazone was not associated with an increased risk of bladder cancer in any analysis, suggesting the risk is drug specific and not a class effect.

KBF holds a Canadian Institutes of Health Research new investigator award, and RWP is supported by a Chercheur-National Award of the Fonds de Recherche du Quebec-Santé (FRQS; Quebec Foundation for Health Research).

Contributors: MT, KBF, OHY, RWP, and LA conceived and designed the study. LA acquired the data. MT, HY, and LA carried out the statistical analysis. All authors analysed and interpreted the data. MT drafted the manuscript. All authors critically revised the manuscript for important intellectual content. LA obtained funding. LA supervised the study and is the guarantor.

Funding: This study is funded by the Canadian Institutes of Health Research.

Competing interests: All authors have completed the ICMJE uniform disclosure form at www.icmje.org/coi_disclosure.pdf and declare: this study was funded by the Canadian Institutes of Health Research; no financial relationships with any organisations that might have an interest in the submitted work in the previous three years; no other relationships or activities that could appear to have influenced the submitted work.
Ethical approval: The study protocol was approved by the independent scientific advisory committee of the CPRD (protocol 11_099A) and by the research ethics board of the Jewish General Hospital, Montreal, Canada.

\section{Data sharing: No additional data available.}

Transparency: The lead author (LA) affirms that this manuscript is an honest, accurate, and transparent account of the study being reported; that no important aspects of the study have been omitted; and that any discrepancies from the study as planned (and, if relevant, registered) have been explained.

This is an Open Access article distributed in accordance with the Creative Commons Attribution Non Commercial (CC BY-NC 3.0) license, which permits others to distribute, remix, adapt, build upon this work non-commercially, and license their derivative works on different terms, provided the original work is properly cited and the use is non-commercial. See: http://creativecommons.org/licenses/ by-nc/3.0/.

\section{Kung J, Henry RR. Thiazolidinedione safety. Expert Opin Drug Saf} 2012;11:565-79

2 Dormandy JA, Charbonnel B, Eckland DJ, et al. PROactive Investigators. Secondary prevention of macrovascular events in patients with type 2 diabetes in the PROactive Study (PROspective pioglitAzone Clinical Trial In macroVascular Events): a randomised controlled trial. Lancet 2005;366:1279-89.

3 Turner RM, Kwok CS, Chen-Turner C, Maduakor CA, Singh S, Loke YK. Thiazolidinediones and associated risk of bladder cancer: a systematic review and meta-analysis. Br J Clin Pharmacol 2014;78:258-73.

4 Lewis JD, Ferrara A, Peng T, et al. Risk of bladder cancer among diabetic patients treated with pioglitazone: interim report of a longitudinal cohort study. Diabetes Care 2011;34:916-22.

5 Neumann A, Weill A, Ricordeau P, Fagot JP, Alla F, Allemand H. Pioglitazone and risk of bladder cancer among diabetic patients in France: a population-based cohort study. Diabetologia 2012;55:1953-62.

6 Mamtani R, Haynes K, Bilker WB, et al. Association between longer therapy with thiazolidinediones and risk of bladder cancer: a cohort study. J Natl Cancer Inst 2012;104:1411-21.

7 Azoulay L, Yin H, Filion KB, et al. The use of pioglitazone and the risk of bladder cancer in people with type 2 diabetes: nested case-control study. BMJ 2012;344:e3645.

8 Hsiao FY, Hsieh PH, Huang WF, Tsai YW, Gau CS. Risk of bladder cancer in diabetic patients treated with rosiglitazone or pioglitazone: a nested case-control study. Drug Saf 2013;36:643-9.

9 Fujimoto K, Hamamoto Y, Honjo S, et al. Possible link of pioglitazone with bladder cancer in Japanese patients with type 2 diabetes. Diabetes Res Clin Pract 2013;99:e21-3.

10 Jin SM, Song SO, Jung CH, et al. Risk of bladder cancer among patients with diabetes treated with a $15 \mathrm{mg}$ pioglitazone dose in Korea: a multi-center retrospective cohort study. J Korean Med Sci 2014;29:238-42

11 Chang $\mathrm{CH}$, Lin JW, Wu LC, Lai MS, Chuang LM, Chan KA. Association of thiazolidinediones with liver cancer and colorectal cancer in type 2 diabetes mellitus. Hepatology 2012;55:1462-72.

12 Tseng CH. Pioglitazone and bladder cancer: a population-based study of Taiwanese. Diabetes Care 2012;35:278-80.

13 Song SO, Kim KJ, Lee BW, Kang ES, Cha BS, Lee HC. The risk of bladder cancer in korean diabetic subjects treated with pioglitazone. Diabetes Metab / 2012;36:371-8

14 Wei L, MacDonald TM, Mackenzie IS. Pioglitazone and bladder cancer: a propensity score matched cohort study. BrJ Clin Pharmacol 2013;75:254-9.

15 Vallarino C, Perez A, Fusco G, et al. Comparing pioglitazone to insulin with respect to cancer, cardiovascular and bone fracture endpoints, using propensity score weights. Clin Drug Investig 2013;33:621-31.

16 Balaji V, Seshiah V, Ashtalakshmi G, Ramanan SG, Janarthinakani M. A retrospective study on finding correlation of pioglitazone and incidences of bladder cancer in the Indian population. Indian I Endocrinol Metab 2014;18:425-7.

17 Kuo HW, Tiao MM, Ho SC, Yang CY. Pioglitazone use and the risk of bladder cancer. Kaohsiung / Med Sci 2014:30:94-7.

18 Lee MY, Hsiao PJ, Yang YH, Lin KD, Shin SJ. The association of pioglitazone and urinary tract disease in type 2 diabetic Taiwanese bladder cancer and chronic kidney disease. PLoS One 2014;9:e85479.

19 Levin D, Bell S, Sund R, et al. Scottish Diabetes Research Network Epidemiology Group Diabetes and Cancer Research Consortium. Pioglitazone and bladder cancer risk: a multipopulation pooled, cumulative exposure analysis. Diabetologia 2015:58:493-504

20 Lewis JD, Habel LA, Quesenberry CP, et al. Pioglitazone Use and Risk of Bladder Cancer and Other Common Cancers in Persons With Diabetes. JAMA 2015;314:265-77. 
21 Herrett E, Gallagher AM, Bhaskaran K, et al. Data Resource Profile: Clinical Practice Research Datalink (CPRD). Int J Epidemiol 2015;44:827-36

22 Khan NF, Harrison SE, Rose PW. Validity of diagnostic coding within the General Practice Research Database: a systematic review. Br J Gen Pract 2010;60:e128-36.

23 Williams T, van Staa T, Puri S, Eaton S. Recent advances in the utility and use of the General Practice Research Database as an example of a UK Primary Care Data resource. Ther Adv Drug Saf 2012;3:89-99.

24 Boggon R, van Staa TP, Chapman M, Gallagher AM, Hammad TA Richards MA. Cancer recording and mortality in the General Practice Research Database and linked cancer registries. Pharmacoepidemiol Drug Saf 2013;22:168-75.

25 Heinzl H, Kaider A, Zlabinger G. Assessing interactions of binary time-dependent covariates with time in cox proportional hazards regression models using cubic spline functions. Stat Med 1996;15:2589-601.

26 Romano P, Roos L, Jollis J. Response: Further evidence concerning the use of a clinical comorbidity index with ICD-9-CM administrative data. J Clin Epidemiol 1993;46:1085-90.

27 Fine JP, Gray RJ. A proportional hazards model for the subdistribution of a competing risk. J Am Stat Assoc 1999;94:496-509.

28 EMA. European Medicines Agency recommends new contraindications and warnings for pioglitazone to reduce small increased risk of bladder cancer. http://www.emaeuropaeu/ema/ indexjsp?curl=pages/medicines/human/public health alerts $/ 2011 / 07 /$ human pha detail 000033isp\& mid $=$ \& source $=$ homeMedSearch\&category=human\&jsenabled $=$ true [Accessed 15 Aug 2015] 2011.

29 Schafer IL. Analysis of incomplete multivariate data. Chapman and Hall, 1997.

30 Rubin DB. Multiple imputations for nonresponse in surveys.John Wiley \& Sons, 1987.

31 Schneeweiss S. Sensitivity analysis and external adjustment for unmeasured confounders in epidemiologic database studies of therapeutics. Pharmacoepidemiol Drug Saf 2006;15:291-303.

32 Schneeweiss S, Rassen JA, Glynn RJ, Avorn J, Mogun H, Brookhart MA. High-dimensional propensity score adjustment in studies of treatment effects using health care claims data. Epidemiology 2009;20:512-22.

33 Ray WA. Evaluating medication effects outside of clinical trials: new-user designs. Am J Epidemiol 2003;158:915-20.
34 Suissa S. Immortal time bias in pharmaco-epidemiology. Am J Epidemiol 2008;167:492-9.

35 Kernan WN, Viscoli CM, Furie KL, et al. Pioglitazone after Ischemic Stroke or Transient Ischemic Attack. N Engl J Med 2016, published online 17 Feb.

36 Viscoli CM Brass LM Carolei A et al. IRIS Trial investigators. Pioglitazone for secondary prevention after ischemic stroke and transient ischemic attack: rationale and design of the Insulin Resistance Intervention after Stroke Trial. Am Heart J 2014:168:823-9.e6.

37 Ryder RE. Pioglitazone: reports of its death are greatly exaggerated - it is alive and ready to resume saving lives. Diabet Med 2015;32:e9-15.

38 Gale EA. Pioglitazone: are rumours of its death exaggerated?Diabet Med 2015;32:431-7.

39 Iles A. NICE issues guidance on diabetes treatments. BMJ 2003;327:520.

40 Hillaire-Buys D, Faillie JL, Montastruc JL, Petit P. Stay vigilant: a glitazone (pioglitazone) can hide a glitazar!Eur J Clin Pharmacol 2012;68:1681-3.

41 Orasanu G, Ziouzenkova O, Devchand PR, et al. The peroxisome proliferator-activated receptor-gamma agonist pioglitazone represses inflammation in a peroxisome proliferator-activated receptor-alphadependent manner in vitro and in vivo in mice. J Am Coll Cardiol 2008;52:869-81

42 Oleksiewicz MB, Southgate J, Iversen L, Egerod FL. Rat urinary bladde carcinogenesis by dual-acting PPARalpha + gamma agonists. PPAR Res 2008;2008:103167.

43 Egerod FL, Brünner N, Svendsen JE, Oleksiewicz MB. PPARalpha and PPARgamma are co-expressed, functional and show positive interactions in the rat urinary bladder urothelium. J Appl Toxicol 2010;30:151-62

44 Egerod FL, Svendsen JE, Hinley J, et al. PPAR alpha and PPAR gamma coactivation rapidly induces Egr-1 in the nuclei of the dorsal and ventral urinary bladder and kidney pelvis urothelium of rats. Toxicol Pathol 2009;37:947-58.

Web extra: Supplementary material 